\title{
Combining clinical and dosimetric features in a PBS proton therapy cohort to develop a NTCP model for radiation- induced optic neuropathy
}

\section{Short Running Title: RION NTCP modelling for proton therapy}

\footnotetext{
$\underline{\text { A Köthe }}^{1,2}$, MSc, P van Luijk ${ }^{3,4}, \mathrm{PhD}, \mathrm{S}$ Safai ${ }^{1}, \mathrm{PhD}, \mathrm{M}$ Kountouri ${ }^{5}, \mathrm{MBChB}, \mathrm{MSc}, \mathrm{AJ}$ Lomax ${ }^{1,2}$, PhD, DC Weber ${ }^{1,6,7}, \mathrm{MD}, \mathrm{G}$ Fattori ${ }^{1}, \mathrm{PhD}$

${ }^{1}$ Center for Proton Therapy, Paul Scherrer Institute, Villigen, Switzerland

${ }^{2}$ Department of Physics, ETH-Hönggerberg, Zurich, Switzerland

${ }^{3}$ Department of Biomedical Sciences of Cells and Systems, Section Molecular Cell Biology, University Medical Center Groningen, University of Groningen, Groningen, Netherlands

${ }^{4}$ Department of Radiation Oncology, University Medical Center Groningen, University of Groningen, Groningen, Netherlands.

${ }^{5}$ Radiation Oncology Department, University Hospital of Geneva, Geneva, Switzerland

${ }^{6}$ Radiation Oncology Department, Inselspital Universitätsspital Bern, Bern, Switzerland

${ }^{7}$ Radiation Oncology Department, University Hospital of Zurich, Zurich, Switzerland
}

\section{Author Information:}

\section{Corresponding Author}

Andreas Köthe

WBBB 115

Paul Scherrer Institute

Forschungsstrasse 111

5323 Villigen

Switzerland

andreas.koethe@psi.ch

+41563103732

Author responsible for statistical analysis

Andreas Köthe

WBBB 115

Paul Scherrer Institute

Forschungsstrasse 111

5323 Villigen

Switzerland

This document is the accepted manuscript version of the following article:

Köthe, A., van Luijk, P., Safai, S., Kountouri, M., Lomax, A. J., Weber, D. C., \& Fattori, G. (2021). Combining clinical and dosimetric features in a PBS proton therapy cohort to develop a NTCP model for radiation-induced optic neuropathy. International Journal of Radiation oncology Biology Physics. https://doi.org/10.1016/j.ijrobp.2020.12.052 
andreas.koethe@psi.ch

+41563103732

\section{Conflict of interest}

The authors declare no conflict of interest.

\section{Funding Statement}

This project was supported by the grant \#2018-223 of the Strategic Focal Area "Personalized Health and Related Technologies (PHRT)" of the ETH Domain.

\section{Data Sharing Statement}

Research data are not available at this time due to sensible patient information.

\section{Acknowledgments}

We would furthermore like to acknowledge everyone participating in the collection of this proton database. 


\section{Short running title: RION NTCP modelling for proton therapy}

\section{Abstract}

Purpose: Radiation-induced optic neuropathy (RION) is a rare, yet severe complication following radiation therapy for brain, head and neck or skull-base tumors. While several risk factors such as age, metabolic syndrome and delivered dose have been identified, we aimed at expanding the understanding of the mechanisms of interplay regarding dosimetry and patient variables leading to the onset of RION with a focus on proton therapy.

Methods and Materials: In this retrospective study we have investigated proton-specific risk factors by comparing common phenomenological NTCP models with a multivariate analysis that includes clinical features on a cohort of skull-base and head and neck cancer patients treated with pencil beam scanning (PBS).

Results: Although predictive power of the Lyman-Kutcher-Burman and Poisson models was limited for this dataset, the addition of clinical variables such as age, tumor involvement, hypertension or gender remarkably increased model performance.

Conclusion: Based on our assessment, the maximum dose in the optical apparatus is confirmed the most intuitive risk factor. However, above a certain dose threshold, clinical patient characteristics are the deciding factors for the onset of RION. Interestingly, we observed a tendency towards a volume effect that, if confirmed, would imply a benefit for high precision radiotherapy techniques such as proton therapy for the treatment of patients with high clinical risk for RION. 


\section{Introduction}

Radiation-induced optic neuropathy (RION) is an acute late normal tissue complication that causes sudden and potentially irreversible, uni- or bilateral blindness in head and neck or central nerve system cancer patients treated with radiation therapy ${ }^{1,2}$. Even though this side effect is rare, it has detrimental impact on the quality of life of patients post-treatment, with currently limited available therapeutic armamentarium to reverse the symptoms ${ }^{3}$. In addition to high dose to the optic apparatus the most commonly reported risk factors for RION toxicity following radiation therapy are gender, age, hypertension, chemotherapy, diabetes and compression of the optic apparatus ${ }^{1,4-14}$. Among these, the dose distribution is arguably the most controllable variable, especially when high precision radiotherapy techniques are available. While several Normal Tissue Complication Probability (NTCP) models for RION based on dose alone have been proposed ${ }^{4,6,14-16}$, and even indications of a volume effect reported ${ }^{8,10,17}$, the only clinically used constraint for the optic structures is typically the maximum dose delivered to the optic apparatus ${ }^{7,18,19}$. Indeed, most classical NTCP models, such as Lyman-Kutcher-Burman (LKB) $)^{16,20,21}$ or Poisson ${ }^{15,22}$ only take into account dosimetric information, even though these could be extended to also account for clinical risk factors ${ }^{6,23-25}$. In addition, published NTCP models for RION are almost exclusively derived from cohorts of patients treated using photons or photon-proton mixed treatments. However, with almost no exit dose after the Bragg peak, resulting in significant reductions in integral dose, proton therapy has a dosimetric advantage compared to conventional photon therapy ${ }^{26,27}$, as well as having sufficiently different dose distributions to make the validity of NTCP models derived from photon therapy questionable when applied to this modality. To the best of our knowledge however, the existing NTCP models have not been tested against 
proton therapy outcomes, nor have NTCP models for RION been developed exclusively from proton therapy outcomes data.

In this work, we test existing, photon derived NTCP models for RION, and develop new models based on previously published clinical results for pencil-beam scanning (PBS) proton therapy ${ }^{28}$. First, the performance of two published RION NTCP models for predicting RION in PBS proton therapy patients has been evaluated. Second, new, logistic regression NTCP models have been developed that combine clinical and dosimetric parameters in order to optimize quantitative risk estimation performance and potentially reveal specific factors associated with RION onset after proton therapy.

\section{Materials and Methods}

The study workflow is visualized in Figure 1. A large cohort of skull-base and head and neck tumor patients treated with proton therapy has been used to benchmark different NTCP models. Initially, the performance of classical NTCP models for RION has been evaluated. In a second step, comprehensive logistic regression models have been developed which take into account both patient specific clinical parameters and dosimetry.

\section{A. Data collection}

Data from 216 adult skull-base and head and neck cancer patients treated with PBS proton therapy between 1999 and 2014 have been considered, a detailed description of which has been published elsewhere ${ }^{28}$ (supplement $A 1, A 2$ ). RION occurred in 14 patients (6.5\%). 
Twenty patients, one with RION, who required re-planning during therapy have however been excluded from our study, due to the uncertainty of accumulating dose on multiple CTs, leaving 196 patients for the analysis. In these remaining patients, there were incidences of RION in 16 structures in 13 patients (6.6\%). Patient age ranged from 18 to 77 years (median follow-up: 5 years) and toxicity was assessed from follow up magnetic resonance (MR) images distinguishing between RION in the chiasm or the left or right optic nerves. All patients included in the cohort received at least $45 \mathrm{~Gy}_{\mathrm{RBE}}\left(\mathrm{D}_{\max }\right)$ to one or more of the optic structures, calculated using a fixed RBE of $1.1^{29}$. Due to mixed fractionation regimes (1.8 2.0 $\mathrm{Gy}_{\mathrm{RBE}} /$ fraction) applied to the patients however, all fraction doses have been converted to a biologically equivalent dose $(\mathrm{BED})^{30}$ of $2 \mathrm{~Gy}_{\mathrm{RBE}}$ per fraction with an $\frac{\alpha}{\beta}$-ratio of $1.07 \mathrm{~Gy}^{31}$ which is in the range of other published values ${ }^{4}$ and within the recommended range for clinical use ${ }^{32}$. Data processing and analysis was done with MATLAB ${ }^{\circledR}$ R2018b (Mathworks Inc, Natick, MA, USA).

\section{B. Classical NTCP models}

In order to evaluate the performance of published NTCP models on our proton cohort, two published models, both developed from conventional photon therapy toxicity data, were selected:

1. The LKB model proposed in the QUANTEC review ${ }^{10}\left(n=0.25, m=0.14, \mathrm{TD}_{50}=70.125\right.$ Gy).

2. A Poisson parametrisation reported by Moiseenko et al. ${ }^{15}\left(\gamma=0.99, \mathrm{TD}_{50}=102 \mathrm{~Gy}\right)$. 
Risk predictions, derived from the applied proton dose distributions and for each of the chiasm and left and right optic nerves, have been calculated using both models.

\section{C. Multi-parametric logistic regression modelling.}

The workflow for development of these models is shown in the right arm of Figure 1. In total, 7 clinical and 166 dosimetric parameters were initially considered. The clinical parameters include age, gender (G), hypertension (HT), hypercholesterolemia, smoking, diabetes and tumor involvement (TI). Tumor involvement was positive if the tumor was in direct contact with any of the optical structures. For all structures within the optic apparatus minimum, mean and maximum doses were calculated as well as a range of $D_{x}(x=1-99 \%)$ and $V_{y}\left(1-64 G y_{R B E}\right)$ metrics, where $D_{x}$ is the minimum dose given to $x \%$ of the structure's volume and $V_{y}$ is the volume in $\%$ that gets at least $y$ Gy $y_{R B E}$ dose ${ }^{33}$.

Strongly correlated parameters can affect the robustness of parameters in a logistic regression model. Therefore, statistically independent variables were selected by identifying those with the highest correlation with RION, whilst removing all other parameters with high cross-correlation, determined using Spearman's rank correlation coefficient (SRCC) ${ }^{34}$. Variables with a SRCC $>0.8$ were considered to be cross-correlated. From this remaining set, the model parameters were selected into the logistic regression model by forward stepwise selection using the stepwiseg/m function from MATLAB ${ }^{\circledR}$. In addition to evaluating each variables' statistical power by the stepwise selection process, we assessed parameter selection robustness by creating 5000 bootstrap $^{35}$ samples of the dataset and by performing 
stepwise logistic regression on each of them. Based on parameter selection frequency, a range of logistic regression models were built, each based on between 1 and 5 of the most prevalent parameters from the bootstrap analysis.

\section{C.IV. Performance Evaluation}

Prediction performance of each model was evaluated using a leave-one-out cross-validation (LOO-CV) technique. A total of 196 models were fitted for each model, each with one of the cohort patients removed from the modelling process. For each model, the structures and data from the 'left-out' patient were then used to assess the predictive performance. Predicted values of each model developed in the LOO-CV process were compared to the observed toxicities. Metrics for performance evaluation included the binary cross-entropy (CE), defined as the negative log-likelihood scaled by the number of samples, allowing to compare the likelihood of our data fitting the different models. Receiver operating characteristic (ROC) curves and the area under the curve (AUC-)ROC value were employed

to gain insight into classification ability and a Hosmer-Lemeshow $(\mathrm{HL})^{36}$ test statistic and calibration curve were calculated to evaluate model calibration. Statistical significance threshold for the HL evaluation was set to 0.05 . To allow comparison between all built models, only predictions for patients with information about hypertension were considered for performance assessment. The reported model parameters are however based on a fit to all available data to maximise the usage of the data. 
The results of NTCP models, including logistic regression models, are necessarily structure based and provide a probability of toxicity in individual structures, rather than the total risk of toxicity to the patient. Therefore, a final patient-wise RION prediction is obtained by combining the sub-volume risks and using the organ seriality of the optic apparatus ${ }^{10}$. Under this assumption, the probability that a patient will be affected by RION is given by

$$
N T C P_{R I O N}=1-\left(1-N T C P_{C H I}\right)\left(1-N T C P_{O N L}\right)\left(1-N T C P_{O N R}\right)
$$

where $N T C P_{s t r}$ is the complication probability predicted by the model for structure str including the chiasm ( $\mathrm{CHI})$ and the left (ONL) and right (ONR) optic nerves. After selection of the best structure-wise performing models, a patient-wise performance evaluation was performed using the same metrics and LOO-CV predictions.

Due to the limited amount of data and their imbalanced nature, it is necessary to assess model order. In order to be able to select a reasonable number of model parameters ${ }^{23}$, the Akaike information criterion $(\mathrm{AIC})^{37}$ and the Bayes information criterion $(\mathrm{BIC})^{38}$ were calculated for all logistic regression models with varying number of parameters.

\section{Results}

\section{A. Performance of the published models and the reparametrized Poisson}


To test whether published models describe the present data, their classification and calibration performance was assessed in our cohort. Table 1 shows the CE, the AUC-ROC and the HL performance evaluations. Performance metrics of the reference models from the literature are comparable to each other, with AUC-ROC values of approximatively 0.5 showing their limited ability to classify patient as RION or non-RION in the present data. In

addition, the $\mathrm{HL}$-value indicates a poor calibration to the data $\left(\mathrm{p}_{\mathrm{HL}} \ll 0.05\right)$ for the QUANTEC model with borderline calibration significance $\left(p_{H L}=0.06-0.08\right)$ for the Moiseenko et al. model. In conclusion, published dosimetry-based models do not describe toxicity in our cohort and new models are needed.

\section{B. Development of NTCP models based on dosimetric and clinical}

\section{parameters}

Due to the poor performance of the published classical models, we aimed at building new NTCP models integrating clinical information in addition, with the aim of improving model performance by making use of reported clinical risk factors.

\section{B.I. Multicollinarity parameter removal}

Cross-correlation analysis among the seven clinical parameters showed that there were no significant correlations (SRCC $<0.8$ ), so all were included in the logistic regression modelling. However, age and hypertension were found to be the most correlated to toxicity (SRCC = 0.18 , figure $2 A)$. On the other hand, the majority of dosimetric parameters were strongly 
correlated to each other (Figure $2 \mathrm{~B},|\mathrm{SRCC}|>0.8$ ) but only marginally to RION (Figure $2 \mathrm{~A}$, $|\mathrm{SRCC}|<0.05)$. Interestingly, the dosimetric parameter with the highest correlation to RION was $\mathrm{V}_{1}(\mathrm{SRCC}=0.072)$ closely followed by $\mathrm{V}_{64}$. Based on these results, age, gender, diabetes, hypertension (HT), hypercholesterolemia, smoking, and tumor involvement (TI) were all considered for the logistic regression, together with $V_{1}, V_{64}, V_{51}, V_{61}, D_{1}, V_{12}$ and $V_{60}$, in descending order of correlation to toxicity.

\section{B.II Bootstrapping and parameter selection}

Age (selection frequency $(S F)=90 \%$ ) was found to be the strongest predictor for RION, followed by tumor involvement ( $\mathrm{SF}=60 \%$ ), hypertension $(\mathrm{SF}=43 \%)$, gender $(\mathrm{SF}=32 \%$ ), smoking $(S F=29 \%)$ and $V_{1}(S F=28 \%)$. Frequency of variable selection in the bootstrapped samples (a surrogate of their significance for toxicity prediction) is shown in Figure $2 \mathrm{C}$ for all variables. Among them, the best dosimetric predictor was again $V_{1}$. Figure 3.A shows the lowest AIC and BIC values evaluated over a range of models built varying the number of parameters. Minimum AIC was found at 2 variables, minimum $\mathrm{BIC}$ at 3 . Based on these metrics, the model on our dataset should preferably be built on no more than 2-3 parameters. As such, the two best performing logistic regression models with no more than 3 parameters have been further tested (Table 1, see section 3.D.)

\section{D. Performance Evaluation}


The prediction performance has been evaluated for every model applied to the individual structures, as well as the total risk of RION for the patient. Conventional models from the literature performed worse than both logistic regression models (Table 1 - full data in supplement A4). AUC-ROC values increased to in between 0.78 and 0.82 , CE values to around 0.23 and HL p-values were not significant with 0.14 and 0.48 .

Consistent with the model order estimate based on bootstrapping (Figure 3A), adding the most predictive dosimetric parameter $\left(\mathrm{V}_{1}\right)$ as a fourth parameter worsened the calibration performance and fitting ability compared to the exclusive use of clinical variables (details reported in supplement $A 4)$.

The parameters of the two best performing models are reported in table 2 . The AUC-ROC could be increased from around 0.5 to 0.8 which can be seen in Figure 3.B, emphasizing the distinct difference of the ROC curves for these models compared to the models based on photon data. HL Calibration plots for the patient-wise prediction of the best performing logistic regression models are visualized in figure 3.C and D. The calibration curves seem to indicate an overestimation of NTCP risk for both proposed models, but their respective HL pvalues (0.14 and 0.48$)$ do not indicate statistically insufficient calibration.

\section{Discussion}

In this work, RION incidence was studied in a cohort of skull base and head and neck cancer patients treated with pencil-beam scanning proton therapy. Initially, we assessed the performance of previously-published models based on dosimetric indices only. The study 
however reported rather meagre classification results, with AUC-ROC close to 0.5. Possible reasons these models couldn't be validated include the limited amount of data which the models were fit on as well as substantial population and treatment heterogeneities across the cohorts. This holds true also for QUANTEC ${ }^{10}$ report for the optic structures that, despite being the de-facto reference database, is mostly based on the Emami et al. ${ }^{39}$ review of rather old data and outdated treatment techniques. Regarding the Poisson model ${ }^{15}$, the cohort characteristics, including fractionation and tumor site, closely match our dataset for the large part. Different are however the treatment modality (protons vs. ${ }^{60} \mathrm{Co}, 2,6$ or 17 MeV X-Rays) and an additional inclusion criterion, that we have set at $D_{\max }>45 \mathrm{~Gy}$ in the optic apparatus. In addition, our LKB and Poisson modelling attempts in this cohort led to similar results compared to the two published models in terms of classification ability, supporting the argumentation that in our proton patient cohort RION risk cannot be reliably predicted using dosimetric variables only. In the dose calculation process, it was decided to adopt the clinical standard of a fixed RBE of $1.1^{29}$. It has been shown that this assumption affects the range and biological effectiveness of protons in the patient and is therefore linked to a non-negligible amount of uncertainty. ${ }^{40,41}$ Not taking into account variable RBE, possible variations in proton range ${ }^{42}$ and ultimately within the dose distribution are therefore not detected and could have an impact on the statistical power of dosimetric variables. Especially range uncertainties in combination with the increased end-of-range LET, could impair the estimation of normal tissues dose, such as the optical structures, potentially affecting the modelling outcome of this study. Nevertheless, a fixed RBE is still employed nowadays in proton therapy centres, taking specific countermeasures in planning the treatments to minimize the risk of OAR over-dosage by favouring fields that are tangential to the most critical structures. The discrepancy of classification power between 
the classical dosimetry-based models and the logistic regression ones built on clinical parameters was however shown to be so substantial to reasonably speculate that such uncertainties haven't jeopardized our results. Finally, there is further uncertainty originating from the choice of the $\alpha / \beta$-ratio when converting fractionation schemes from 1.8 to $2 \mathrm{~Gy} /$ fraction. While the sparsity of the data and therefore the accuracy for $\alpha / \beta$-ratios for the optic apparatus is large, employing the values reported by Jiang et al. $(\alpha / \beta=1.6)^{4}$ or Speckter et al. $(\alpha / \beta=1.07)^{31}$ would have led to dosimetric differences of about $1 \%$ with minor impact on the modelling results.

When planning radiotherapy treatments, reducing the maximum dose delivered to serial organs at risk such as the optical apparatus is crucial ${ }^{6,7,18,43}$. Even though dosimetric variables have not been explicitly included in the best models, the maximum dose inclusion criterion of at least $45 \mathrm{~Gy}$ is implicitly contained and leads to an intrinsic bias of RION incidence in this cohort. By setting this dose threshold we made the assumption that no patients are affected with maximum doses lower than $45 \mathrm{~Gy}$. This is certainly true for our cohort, but is also confirmed in the QUANTEC review where a multitude of studies is presented with no patient presenting RION below $55 \mathrm{~Gy}^{10}$. It has to be acknowledged that by leaving out the portion of low-dose patients with arguably negligible likelihood of RION incidence, statistical significance of dosimetric variables was greatly reduced. However, RION incidence is low even in populations with high doses, therefore by pre-selecting the patients, more significance in the model has been attributed to factors influencing the incidence of RION other than high maximum dose, which was assumed to be a pre-requisite for RION incidence. Consequently, we found that clinical factors play an important role. Similarly, in a recent study, $D_{\max }$ was selected together with age and female gender in a 
multivariable RION model based on a mixed proton and photon cohort ${ }^{6}$. Since no incidences are generally reported at lower dose levels $s^{6,10,15}, D_{\max }$ is intuitively considered a risk factor for RION. In addition to that, some authors have discussed the presence of a volume effect for the onset of toxicity in structures belonging to the nervous system ${ }^{44}$, including optic neuropathy ${ }^{8,10,17}$. This is also hinted at by the high ranking of $V_{1}$ following bootstrapping parameter selection in our work (Fig. 2). Although this would need to be confirmed by other groups or by including more data into our analysis, it leads to the intriguing thought that the optic structures could be sensitized by being exposed to a very low-level 'dose-bath' over the whole volume. The reason this has not been observed in photon cohorts could be due to the fact that such a dose bath is almost inevitably present in photon treatments. Interestingly, this could be reduced in the optic structures for certain patients using proton therapy ${ }^{45}$.

Clinical variables are confirmed as key predictors of neuropathy onset in patients receiving at least $45 \mathrm{~Gy}$ to the optic apparatus. Based on the analysis of our proton dataset, we consolidate the literature observations (see supplement A3) that age $\mathrm{a}^{6,7,10,11,19}$, hypertension ${ }^{5,10}$, gender ${ }^{6}$, and tumor involvement ${ }^{1,9}$ are strong risk factors for RION. As is the case in any patient cohort, not every possible variable could be collected or observed. Risk factors, such as prior radiotherapy, chemotherapy or other potentially important factors such as genetic background, were not collected and therefore not included in the modelling. The importance of these variables could unfortunately not be assessed, but could have an impact of the incidence of RION as shown in other studies ${ }^{1,10,14}$. The clinical variables contained in this cohort are integrated in the two best-performing NTCP models to prospectively assess the risk of RION in patients potentially eligible for proton therapy and 
balance it against alternative treatment modalities or planning strategies that might allow dose reduction for high-risk individuals. The prerequisite of a high dose threshold for RION incidence is complemented here by clinical risk factors allowing for significantly improved patient stratification, ultimately opening up the possibility for more specific treatment planning tailored to the patient's risk group. Clinically the presented model allows evaluating the risk of RION if a high dose may be delivered to the optical apparatus. This will help optimizing treatment planning for patients at increased risk due to e.g. age, gender or hypertension by prioritizing sparing the optical structures, by optimizing beam angles, accepting higher doses to other normal tissues or changing treatment modality to e.g. protons. The latter may be especially useful if the negative effect of a dose-bath can be confirmed in external cohorts. Finally, quantification of risk for RION prior to treatment could help to make patients more aware of their specific risk regarding the incidence of RION which will allow for improved patient awareness of radiation side effects. However, until the model is externally validated, it should be applied in a clinical setting only with the utmost care.

Radiation-induced optic neuropathy is a rather rare, yet severe, complication of radiation therapy observed only in about approximately $1 \%{ }^{10}$ of radiotherapy patients across several RION studies. Despite the general agreement on the risk factors, the mechanisms of interplay between radiation dose and patient variables leading to the onset of RION have not been fully understood yet. Arguably, since a significant proportion of patients does not suffer from RION even though several risk factors are present, there are variables unaccounted for that could encompass genetic background or patient-specific radiosensitivity of vasculature or nervous tissue. Furthermore, the low occurrence rate 
challenges probability modelling and the detection of risk factors for RION. An effort for inter-institutional data gathering and cross-validation is recommended to confirm and improve predictive power of NTPC models for RION. In the future, if more and multiinstitutional data were available, one could additionally think about grouping patients according to the detected risk factors and then refit the classical LKB models on the grouped patients. This might improve the reliability of the model predictions significantly.

\section{Conclusion}

We have compared the performance of the widely used Lyman-Kutcher-Burman and Poisson NTCP models with a multivariate analysis that includes clinical parameters for the endpoint of RION in patients treated with PBS proton therapy. Even though the maximal dose in the optic apparatus is the most apparent risk factor, above a certain threshold, patient characteristics such as age, tumour involvement, hypertension and gender are the predominant predictors for the onset of RION. In addition, and despite the assumed serial nature of the optical apparatus, our analysis hints at a sensitisation effect when a low-level dose-bath is applied across the whole optic structure. If confirmed, this could favour high precision radiotherapy techniques such as proton therapy for the treatment of patients with high clinical risk for RION.

\section{Bibliography}

1. Danesh-Meyer H V. Radiation-induced optic neuropathy. J Clin Neurosci.

2008;15(2):95-100. doi:10.1016/J.JOCN.2007.09.004 
2. Aldrees S, Micieli JA. Catastrophic vision loss from radiation-induced optic neuropathy. BMJ Case Rep. 2020;13(2):e233706. doi:10.1136/bcr-2019-233706

3. Indaram M, Ali FS, Levin $\mathrm{MH}$. In search of a treatment for radiation-induced optic neuropathy. Curr Treat Options Neurol. 2015;17(1):1-9. doi:10.1007/s11940-0140325-2

4. Jiang GL, Tucker SL, Guttenberger R, et al. Radiation-induced injury to the visual pathway. Radiother Oncol. 1994;30(1):17-25. doi:10.1016/0167-8140(94)90005-1

5. Doroslovački P, Tamhankar MA, Liu GT, Shindler KS, Ying GS, Alonso-Basanta M. Factors Associated with Occurrence of Radiation-induced Optic Neuropathy at "Safe" Radiation Dosage. Semin Ophthalmol. 2018;33(4):581-588. doi:10.1080/08820538.2017.1346133

6. Li PC, Liebsch NJ, Niemierko A, et al. Radiation tolerance of the optic pathway in patients treated with proton and photon radiotherapy. Radiother Oncol. 2019;131:112-119. doi:10.1016/j.radonc.2018.12.007

7. Noël G, Habrand J-L, Mammar H, et al. Combination of photon and proton radiation therapy for chordomas and chondrosarcomas of the skull base: the Centre de Protonthérapie D’Orsay experience. Int J Radiat Oncol. 2001;51(2):392-398. doi:10.1016/S0360-3016(01)01634-0

8. Bhandare N, Monroe AT, Morris CG, Bhatti MT, Mendenhall WM. Does altered fractionation influence the risk of radiation-induced optic neuropathy? Int J Radiat Oncol. 2005;62(4):1070-1077. doi:10.1016/J.IJROBP.2004.12.009

9. Demizu Y, Murakami M, Miyawaki D, et al. Analysis of Vision Loss Caused by Radiation-Induced Optic Neuropathy After Particle Therapy for Head-and-Neck and Skull-Base Tumors Adjacent to Optic Nerves. Int J Radiat Oncol Biol Phys. 
2009;75(5):1487-1492. doi:10.1016/j.ijrobp.2008.12.068

10. Mayo C, Martel MK, Marks LB, Flickinger J, Nam J, Kirkpatrick J. Radiation DoseVolume Effects of Optic Nerves and Chiasm. Int J Radiat Oncol. 2010;76(3):S28-S35. doi:10.1016/J.IJROBP.2009.07.1753

11. Wang W, Yang H, Guo L, Su H, Wei S, Zhang X. Radiation-induced optic neuropathy following external beam radiation therapy for nasopharyngeal carcinoma: A retrospective case-control study. Mol Clin Oncol. 2016;4(5):868-872. doi:10.3892/mco.2016.787

12. Seibel I, Cordini D, Hager A, et al. Predictive risk factors for radiation retinopathy and optic neuropathy after proton beam therapy for uveal melanoma. Graefe's Arch Clin Exp Ophthalmol. 2016;254(9):1787-1792. doi:10.1007/s00417-016-3429-4

13. Ferguson I, Huecker J, Huang J, McClelland C, Van Stavern G. Risk factors for radiation-induced optic neuropathy: a case-control study. Clin Exp Ophthalmol. 2017;45(6):592-597. doi:10.1111/ceo.12927

14. Milano MT, Grimm J, Soltys SG, et al. Single- and Multi-Fraction Stereotactic Radiosurgery Dose Tolerances of the Optic Pathways. Int J Radiat Oncol. January 2018. doi:10.1016/j.ijrobp.2018.01.053

15. Moiseenko V, Song WY, Mell LK, Bhandare N. A comparison of dose-response characteristics of four NTCP models using outcomes of radiation-induced optic neuropathy and retinopathy. Radiat Oncol. 2011;6:61. doi:10.1186/1748-717X-6-61

16. Burman C, Kutcher GJ, Emami B, Goitein M. Fitting of normal tissue tolerance data to an analytic function. Int J Radiat Oncol. 1991;21(1):123-135. doi:10.1016/03603016(91)90172-Z

17. Martel MK, Sandler HM, Cornblath WT, et al. Dose-volume complication analysis for 
visual pathway structures of patients with advanced paranasal sinus tumors. 1997;38(2):273-284. doi:10.1016/S0360-3016(97)00029-1

18. Weber DC, Rutz HP, Pedroni ES, et al. Results of spot-scanning proton radiation therapy for chordoma and chondrosarcoma of the skull base: The Paul Scherrer Institut experience. Int J Radiat Oncol. 2005;63(2):401-409.

doi:10.1016/J.IJROBP.2005.02.023

19. Weber DC, Chan AW, Lessell S, et al. Visual outcome of accelerated fractionated radiation for advanced sinonasal malignancies employing photons/protons. Radiother Oncol. 2006;81(3):243-249. doi:10.1016/j.radonc.2006.09.009

20. Lyman JT. Complication Probability as Assessed from Dose-Volume Histograms. Radiat Res. 1985;104(2):S13. doi:10.2307/3576626

21. Kutcher GJ, Burman C, Brewster L, Goitein M, Mohan R. Histogram reduction method for calculating complication probabilities for three-dimensional treatment planning evaluations. Int J Radiat Oncol. 1991;21(1):137-146. doi:10.1016/03603016(91)90173-2

22. Warkentin B, Stavrev P, Stavreva N, Field C, Fallone BG. A TCP-NTCP estimation module using DVHs and known radiobiological models and parameter sets. J Appl Clin Med Phys. 2004;5(1):50-63. doi:10.1120/jacmp.v5i1.1970

23. El Naqa I, Bradley J, Blanco Al, et al. Multivariable modeling of radiotherapy outcomes, including dose-volume and clinical factors. Int J Radiat Oncol. 2006;64(4):1275-1286. doi:10.1016/J.IJROBP.2005.11.022

24. Cella L, Liuzzi R, Conson M, D’Avino V, Salvatore M, Pacelli R. Multivariate Normal Tissue Complication Probability Modeling of Heart Valve Dysfunction in Hodgkin Lymphoma Survivors. Int J Radiat Oncol. 2013;87(2):304-310. 
doi:10.1016/j.ijrobp.2013.05.049

25. Cella L, D'Avino V, Palma G, et al. Modeling the risk of radiation-induced lung fibrosis: Irradiated heart tissue is as important as irradiated lung. Radiother Oncol. 2015;117(1):36-43. doi:10.1016/j.radonc.2015.07.051

26. Steneker M, Lomax A, Schneider U. Intensity modulated photon and proton therapy for the treatment of head and neck tumors. Radiother Oncol. 2006;80(2):263-267. doi:10.1016/j.radonc.2006.07.025

27. Cozzi L, Fogliata A, Lomax A, Bolsi A. A treatment planning comparison of 3D conformal therapy, intensity modulated photon therapy and proton therapy for treatment of advanced head and neck tumours. Radiother Oncol. 2001;61(3):287-297. doi:10.1016/S0167-8140(01)00403-0

28.

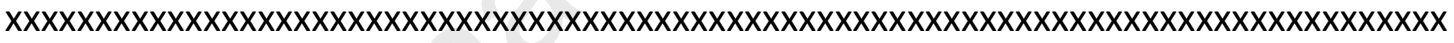

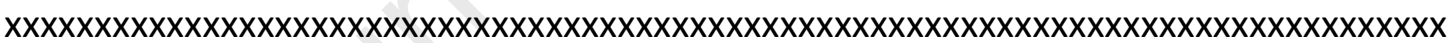
$\operatorname{xxxxxx}$

29. Paganetti H. Relative Biological Effectiveness (RBE) Values for Proton Beam Therapy. Variations as a Function of Biological Endpoint, Dose, and Linear Energy Transfer. Vol 59. IOP Publishing; 2014:R419-R472. doi:10.1088/0031-9155/59/22/R419

30. Fowler JF. The linear-quadratic formula and progress in fractionated radiotherapy. $\mathrm{Br}$ J Radiol. 1989;62(740):679-694. doi:10.1259/0007-1285-62-740-679

31. Speckter H, Santana J, Miches I, et al. Assessment of the alpha/beta ratio of the optic pathway to adjust hypofractionated stereotactic radiosurgery regimens for perioptic lesions. J Radiat Oncol. August 2019:1-11. doi:10.1007/s13566-019-00398-8

32. Joiner M, Kogel A van der. Basic Clinical Radiobiology. 
33. International Commission of Radiation Units and Measurements. Geometric terms and dose, and dose-volume definitions. J ICRU. 2007:83-94.

34. Spearman C. The Proof and Measurement of Association between Two Things. Am J Psychol. 1904;15(1):72. doi:10.2307/1412159

35. Efron B. Bootstrap Methods: Another Look at the Jackknife. Ann Stat. 1979;7(1):1-26. doi:10.1214/aos/1176344552

36. Hosmer DW, Lemesbow S. Goodness of fit tests for the multiple logistic regression model. Commun Stat - Theory Methods. 1980;9(10):1043-1069. doi:10.1080/03610928008827941

37. Akaike H. A new look at the statistical model identification. IEEE Trans Automat Contr. 1974;19(6):716-723. doi:10.1109/TAC.1974.1100705

38. Schwarz G. Estimating the Dimension of a Model. Ann Stat. 1978;6(2):461-464. doi:10.1214/aos/1176344136

39. Emami B, Lyman J, Brown A, et al. Tolerance of normal tissue to therapeutic irradiation. Int J Radiat Oncol Biol Phys. 1991;21(1):109-122. http://www.ncbi.nlm.nih.gov/pubmed/2032882. Accessed July 3, 2019.

40. Resch AF, Landry G, Kamp F, et al. Quantification of the uncertainties of a biological model and their impact on variable RBE proton treatment plan optimization. Phys Medica. 2017;36:91-102. doi:10.1016/j.ejmp.2017.03.013

41. Gutierrez A, Rompokos V, Li K, et al. The impact of proton LET/RBE modeling and robustness analysis on base-of-skull and pediatric craniopharyngioma proton plans relative to VMAT. Acta Oncol (Madr). 2019;58(12):1765-1774. doi:10.1080/0284186X.2019.1653496

42. Grün R, Friedrich $\mathrm{T}$, Krämer $\mathrm{M}$, et al. Physical and biological factors determining the 
effective proton range. Med Phys. 2013;40(11). doi:10.1118/1.4824321

43. Mayo CS, Yao J, Eisbruch A, et al. Incorporating big data into treatment plan evaluation: Development of statistical DVH metrics and visualization dashboards. Adv Radiat Oncol. 2017;2(3):503-514. doi:10.1016/J.ADRO.2017.04.005

44. Van Luijk P, Bijl HP, Konings AWT, Van der Kogel AJ, Schippers JM. Data on dosevolume effects in the rat spinal cord do not support existing NTCP models. Int J Radiat Oncol Biol Phys. 2005;61(3):892-900. doi:10.1016/j.ijrobp.2004.10.035

45. Delaney TF. Proton therapy in the clinic. Front Radiat Ther Oncol. 2011;43:465-485. doi:10.1159/000322511

\section{Figure Captions}

Figure 1: Schematic of the analysis workflow to compare and develop NTCP models for RION. While the classical models incorporate only the dosimetric information (left arm), the developed logistic regression models allow for additional inclusion of a variety of clinical parameters (right arm).

Figure 2: (A) Sperman's rank correlation (SRCC) between RION incidence and a range of dosimetric and clinical parameters. Age and hypertension are the clinical variables most correlated to onset of optic neuropathy. $V_{1}$ is, among the dosimetric variables, the most significant, albeit with a low correlation index equals (SRCC = 0.07). (B) Self-correlation map of all dosimetric variables. (C) Parameter selection frequency from 5000 bootstrap samples of the original dataset. Age, tumor involvement and hypertension were the most frequently chosen variables. The most selected dosimetric parameter was $V_{1}$. 
Figure 3: (A) The minimum AIC and BIC metrics were calculated for each number of model parameters. The metrics start to increase around 2-3 model parameters which means the developed model should not contain more than 3 parameters (B) ROC curves for the 2 best logistic regression models compared to the predictive performance of models based on dosimetry. Classification ability increases significantly when including clinical parameters. (C)+(D) Hosmer-Lemeshow calibration curves for the 2 best logistic regression models. The blue circles represent the observed fraction of one bin of patients plotted against their predicted mean risk of RION.

Table 1: Overview over the performance evaluation metrics for the models based on dosimetry and the 2 best performing logistic regression models including the clinical parameters age, tumor involvement (TI) and hypertension (HT) or gender (G).

Table 2: Model parameters of the two best performing logistic regression NTCP models based on age, tumor involvement (TI) and hypertension (HT) or gender (G). 


\begin{tabular}{ccccc|cccc} 
& \multicolumn{4}{c}{ Structure-wise } & \multicolumn{3}{c}{ Patient-wise } \\
Model & CE & AUC-ROC & $\begin{array}{c}\text { HL test } \\
\text { statistic }\end{array}$ & $\begin{array}{c}\text { HL p- } \\
\text { value }\end{array}$ & CE & AUC-ROC & $\begin{array}{c}\text { HL test } \\
\text { statistic }\end{array}$ & $\begin{array}{c}\text { HL } p-~ \\
\text { value }\end{array}$ \\
\hline Quantec & 0.3337 & 0.5000 & $>>100$ & 0.0000 & 0.5572 & 0.5000 & $>>100$ & 0.0000 \\
Moiseenko et al. Poisson & 0.1438 & 0.5432 & 14.57 & 0.0781 & 0.2890 & 0.5926 & 14.7 & 0.0647 \\
\hline Age + TI + HT & 0.1174 & 0.8116 & 18.76 & 0.0172 & 0.2267 & 0.8168 & 12.2 & 0.1418 \\
Age + TI + G & 0.1175 & 0.7761 & 6.56 & 0.5939 & 0.2346 & 0.7833 & 7.52 & 0.4819
\end{tabular}

Table 1: Overview over the performance evaluation metrics for the models based on dosimetry and the 2 best performing logistic regression models including the clinical parameters age, tumor involvement (TI) and hypertension (HT) or gender (G).

\begin{tabular}{cccccc}
\hline Model & $\boldsymbol{\theta 0}$ (const) & $\boldsymbol{\theta 1}$ (Age) & $\boldsymbol{\theta 2}(\mathrm{TI})$ & $\boldsymbol{\theta 3}(\mathrm{HT} / \mathrm{S})$ & \\
\hline Age + TI + HT & -9.22 & 0.0809 & 1.4593 & 1.0465 & $\boldsymbol{N T C P}(\overrightarrow{\boldsymbol{x}})$ \\
Age $+\mathrm{TI}+\mathrm{G}$ & -10.1802 & 0.0956 & 1.3565 & 0.9192 & $=$
\end{tabular}

Table 2: Model parameters of the two best performing logistic regression NTCP models based on age, tumor involvement (TI) and hypertension (HT) or gender (G). 


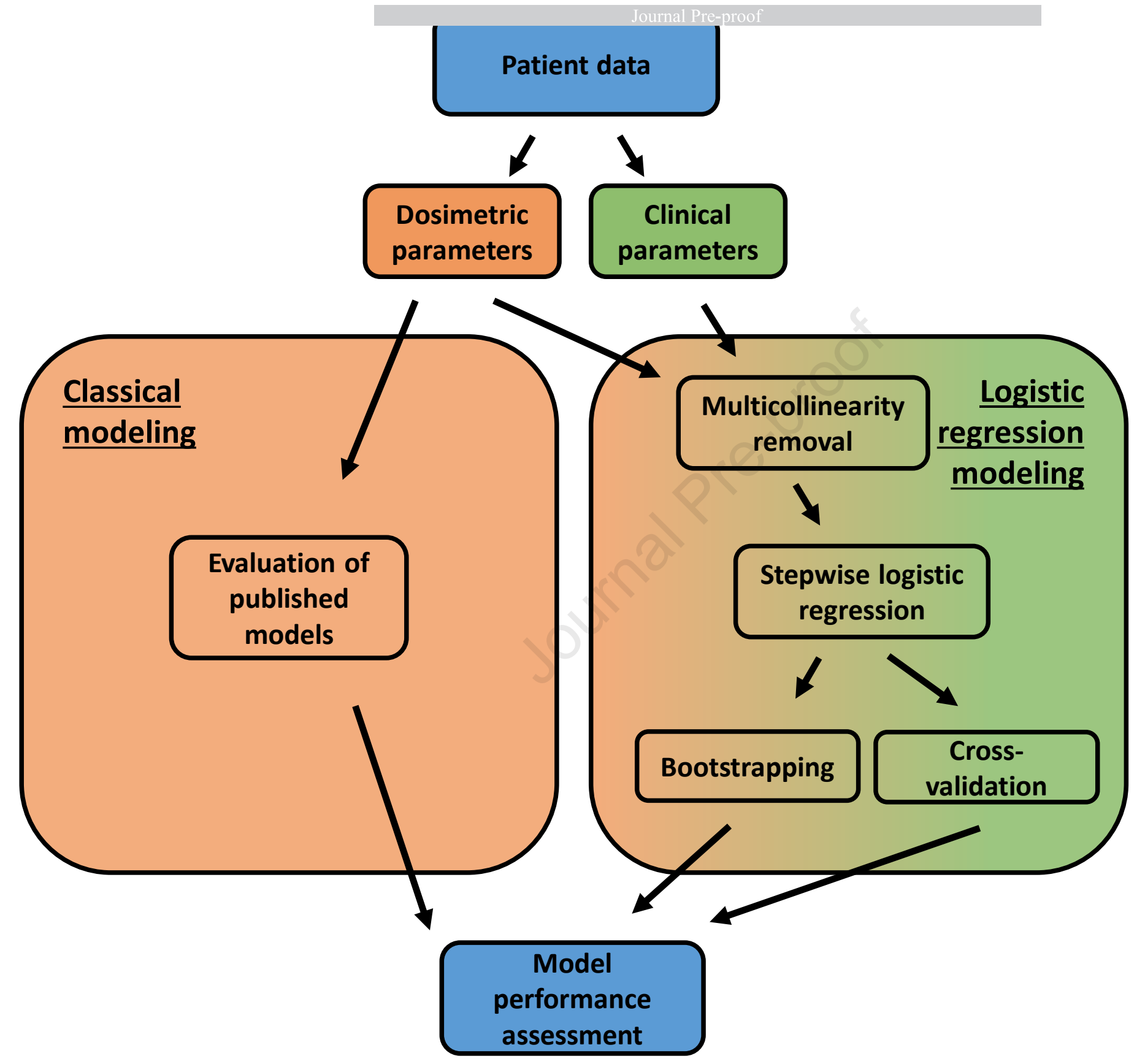




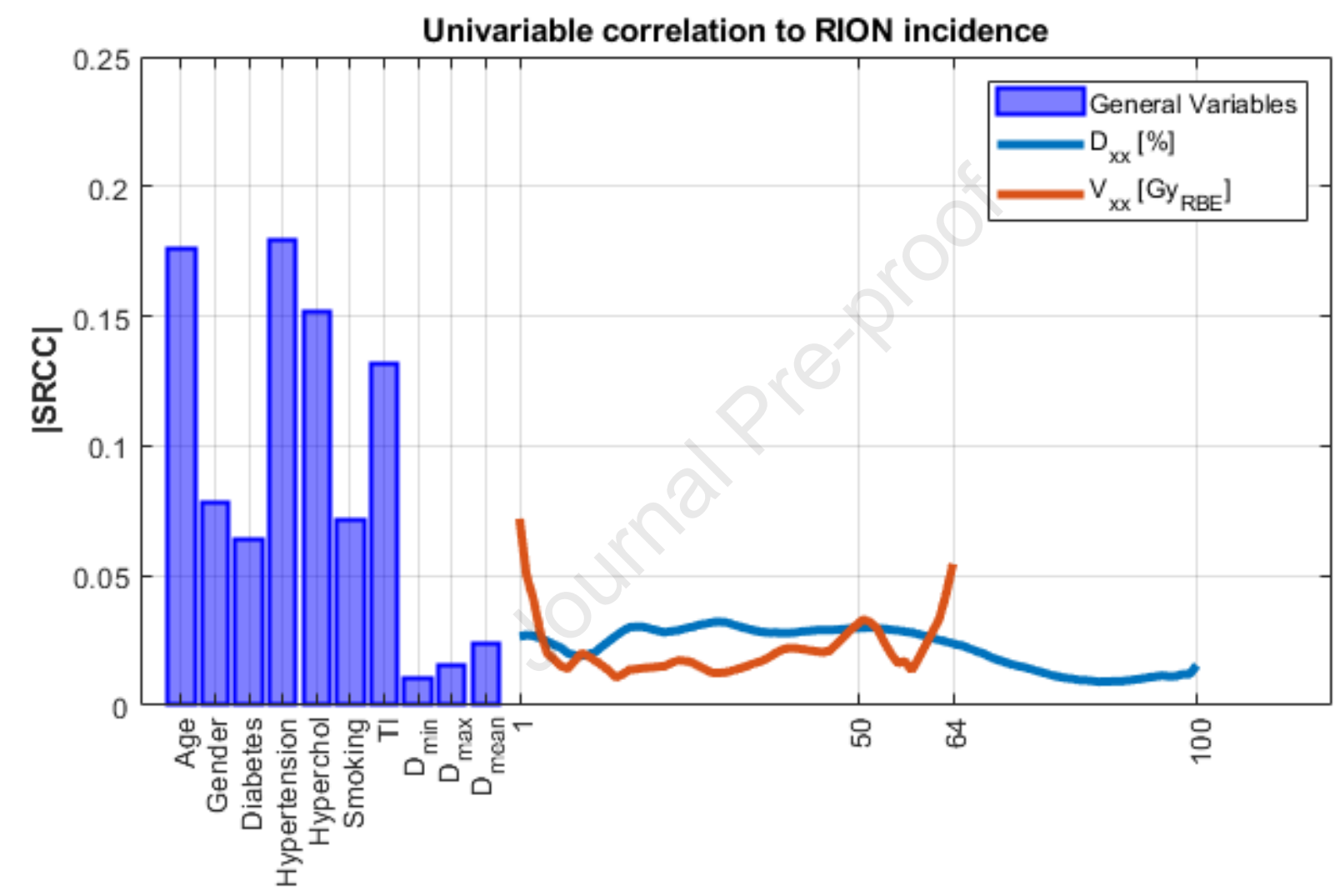




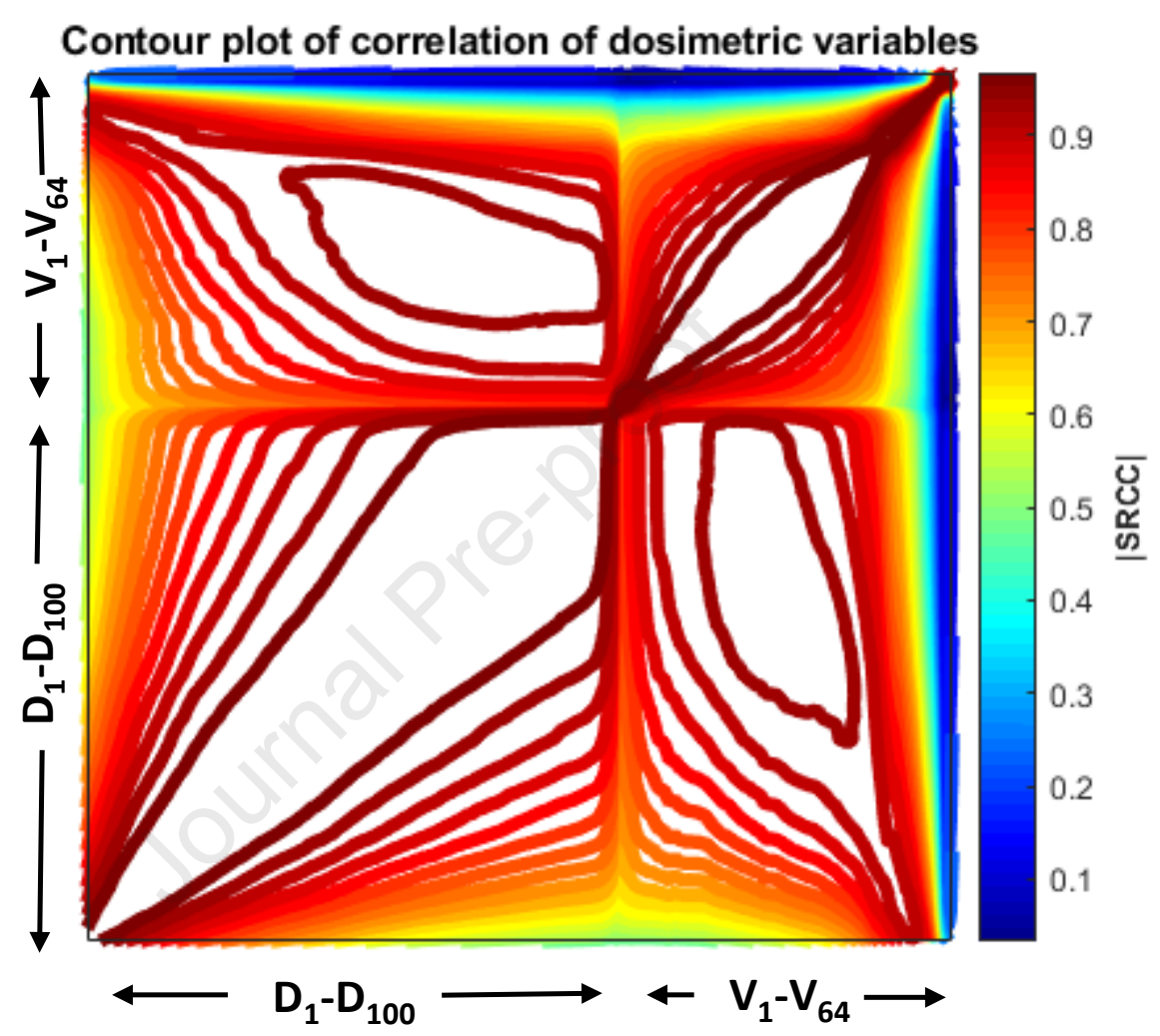


Frequency of variable selection out of 5000 bootstrap samples

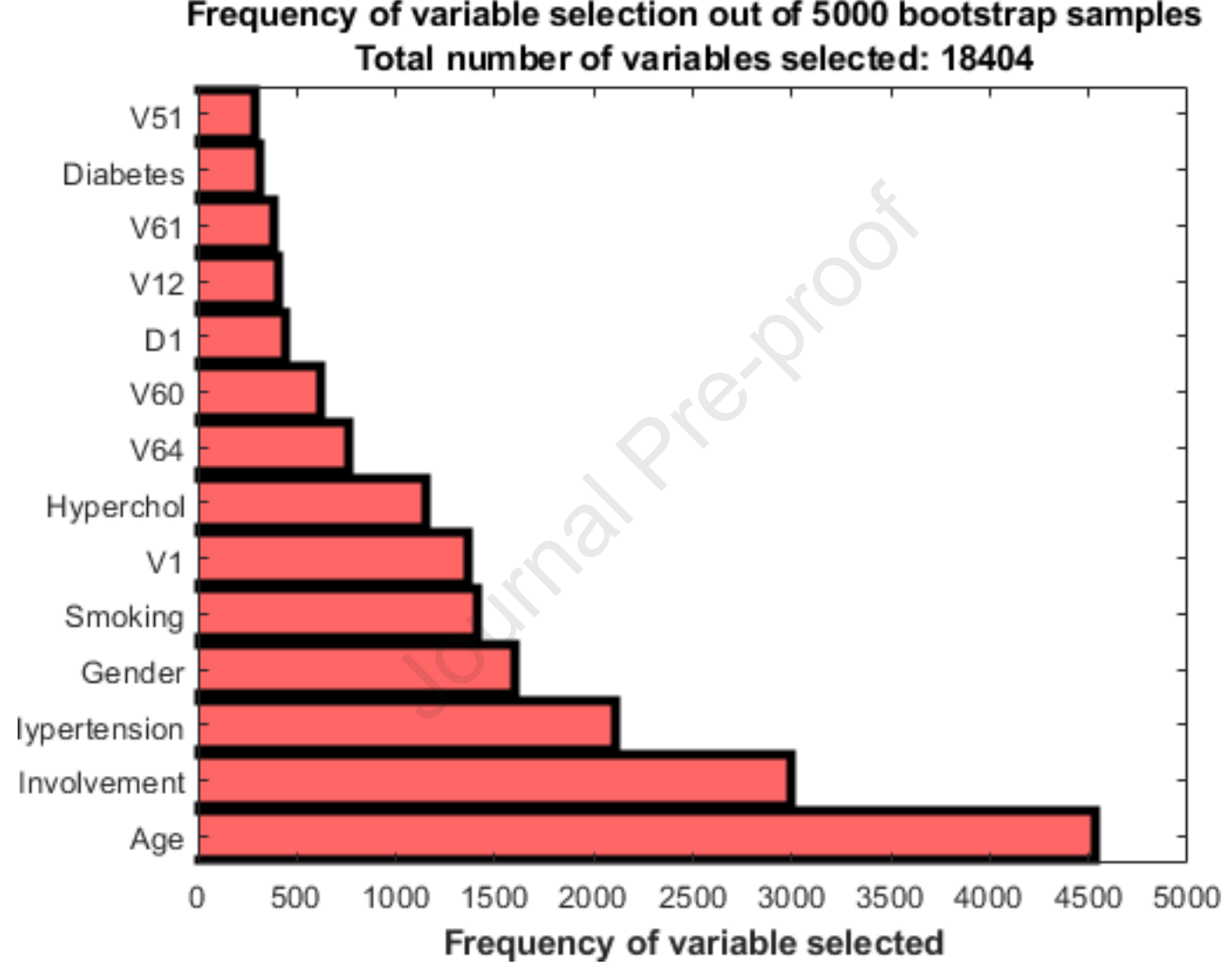




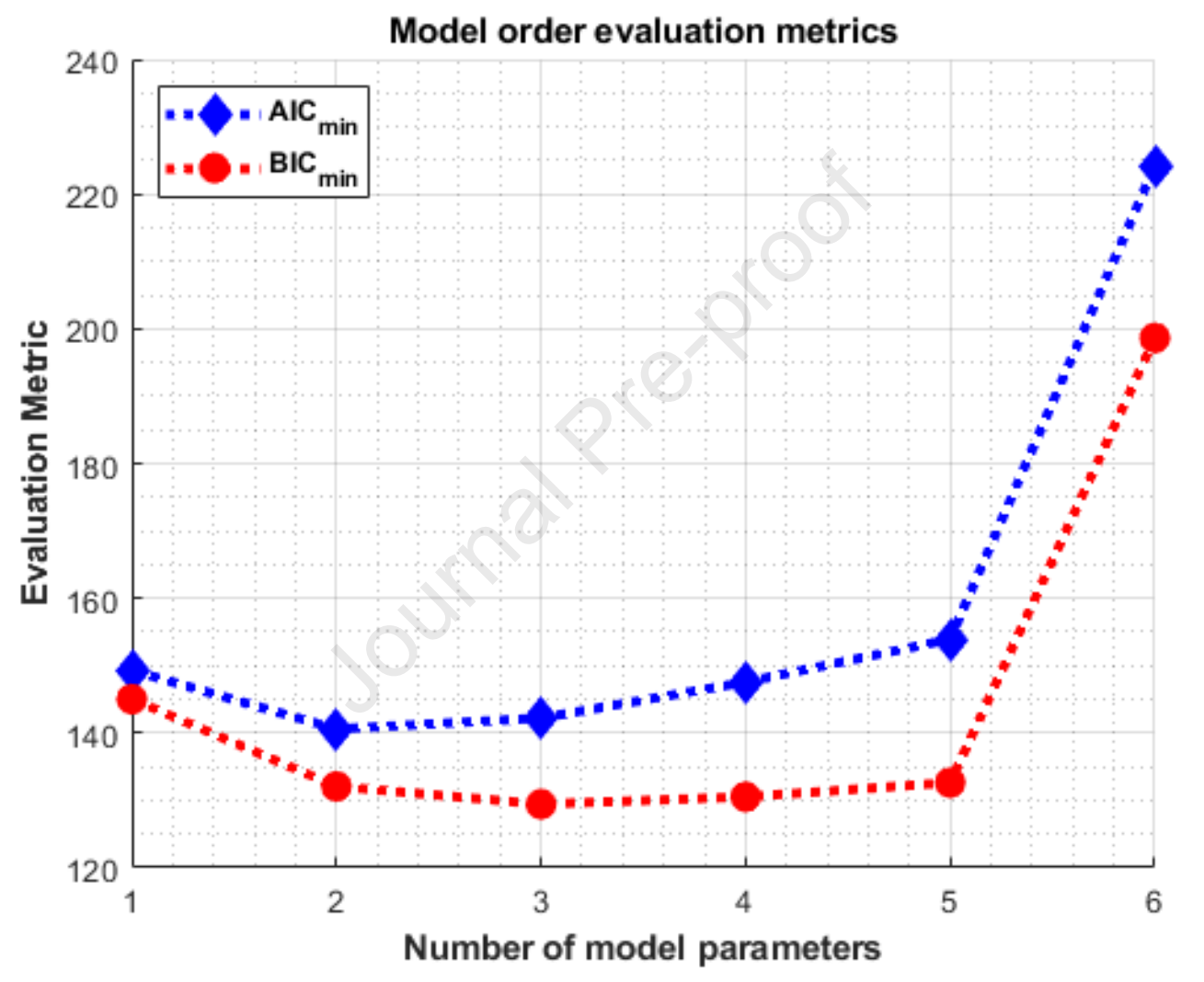




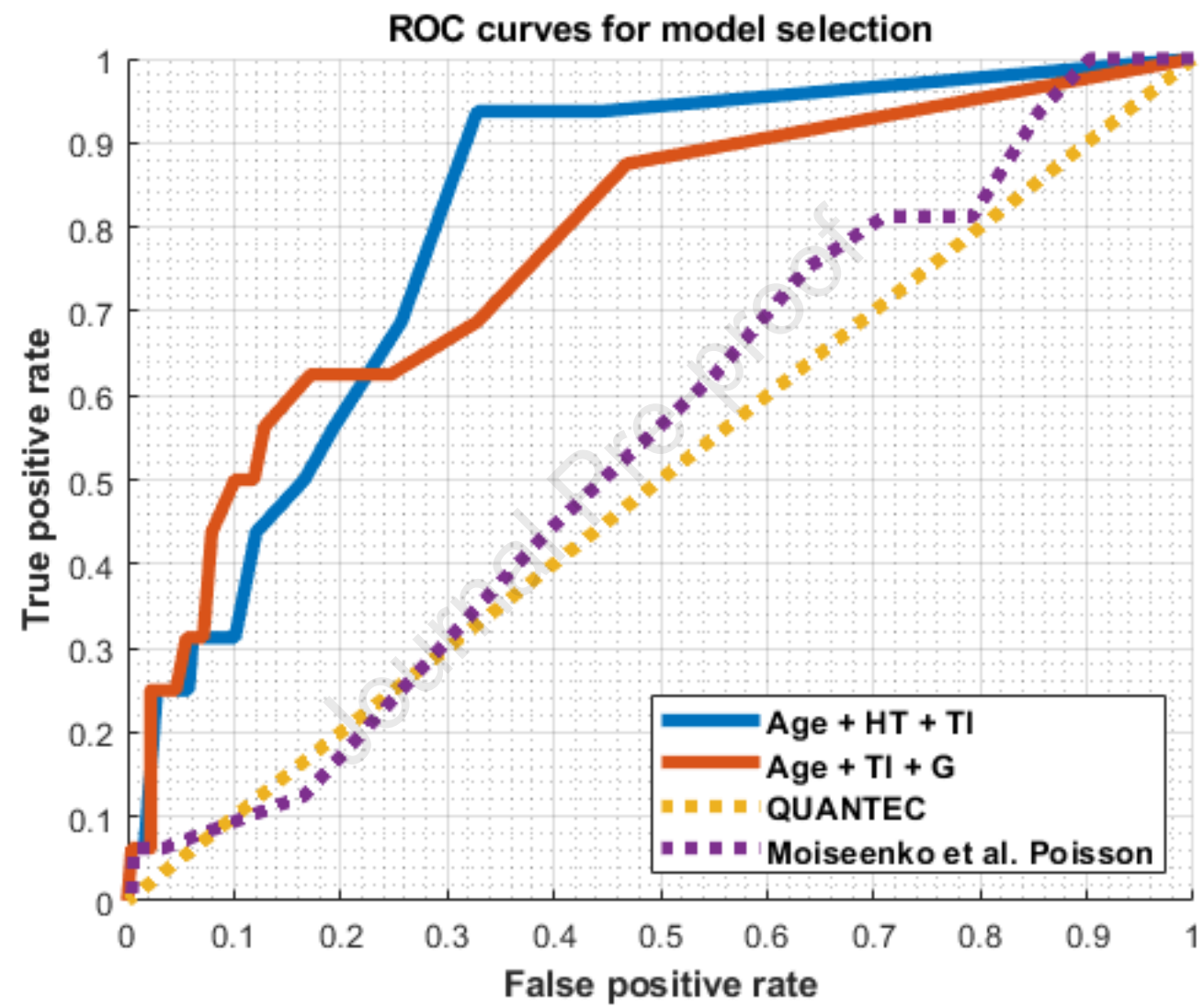




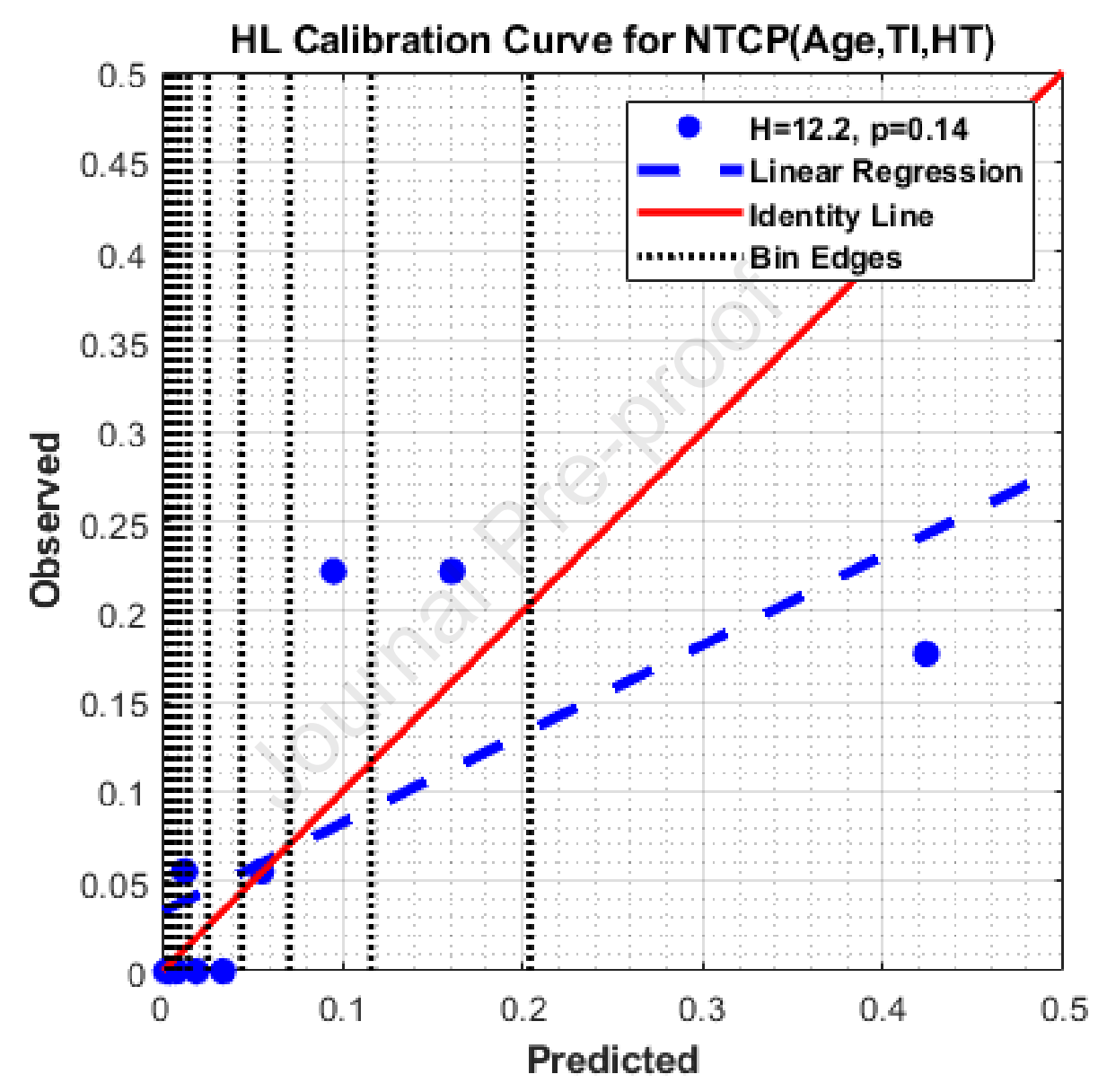




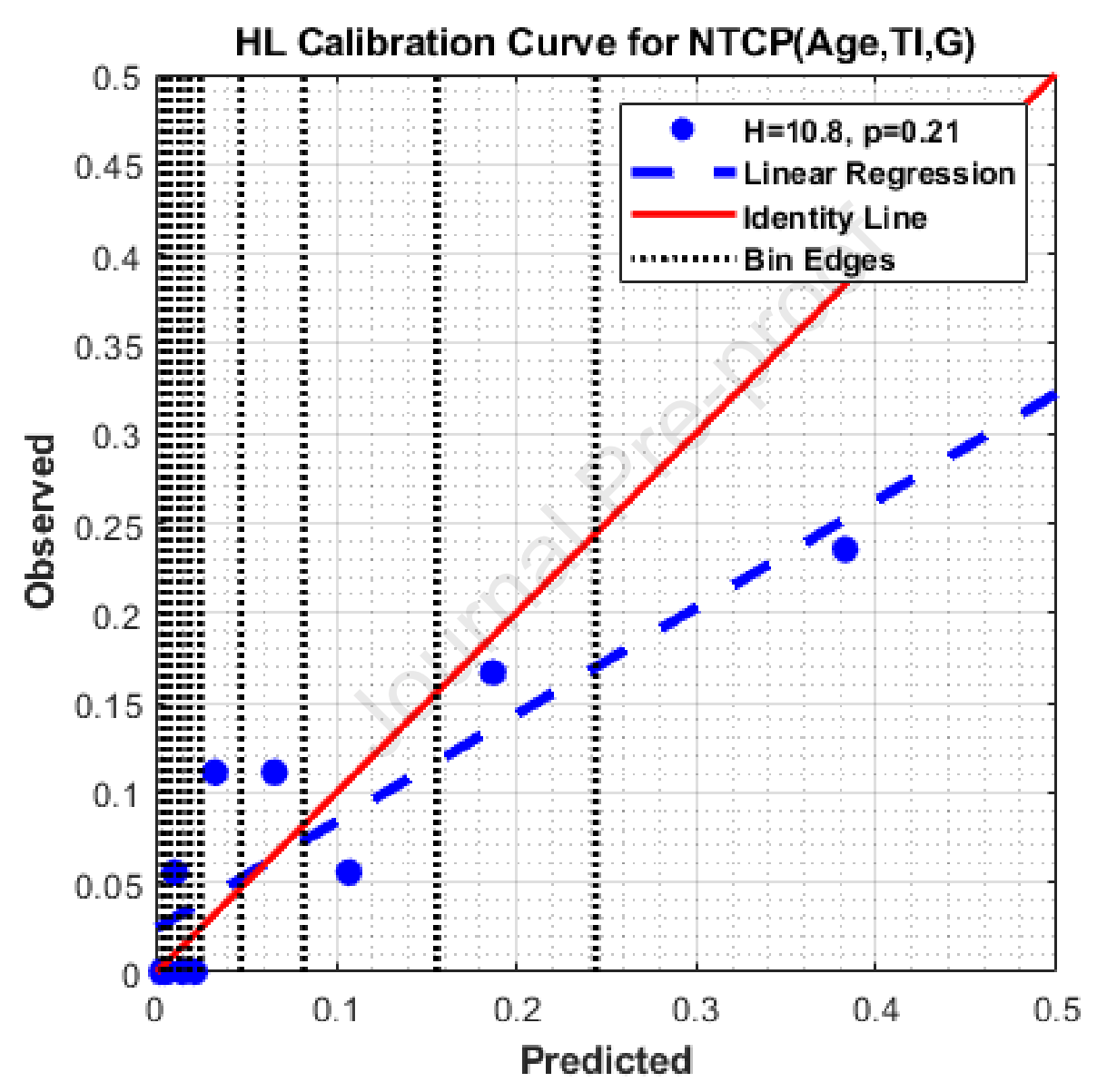

